Received: 5 October 2018

Accepted: 10 December 2018

Published online: 04 February 2019
NTIFIC REP

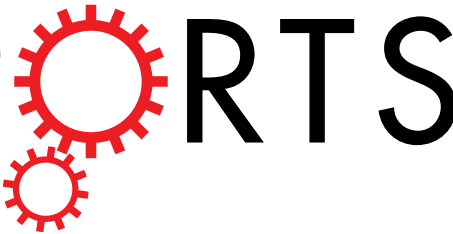

OPEN

\section{Limiting the Spectral Diffusion of Nano-Scale Light Emitters using the Purcell effect in a Photonic- Confined Environment}

\author{
A. Lyasota, C. Jarlov, A. Rudra, B. Dwir \& E. Kapon
}

Partial suppression of the spectral diffusion of quantum dot (OD) excitons tuned to resonance of a nanophotonic cavity is reported. The suppression is caused by the Purcell enhancement of the QD-exciton recombination rate, which alters the rate of charging of the solid-state environment by the QD itself. The effect can be used to spectrally-stabilize solid-state emitters of single photons and other nonclassical states of light.

Electric charging in the environment of nano-scale light emitters can lead to spectral diffusion or intermittency (blinking) of the emission, as observed with nitrogen-vacancy centers in diamond ${ }^{1}$, molecules $^{2}$, carbon nanotubes ${ }^{3}$, semiconductor nanorods ${ }^{4}$ and quantum $\operatorname{dots}^{5,6}$ (QDs) embedded in solid-state matrices. Various aspects $^{7-11}$ and mechanisms $s^{9,12-14}$ of such spectral dynamics were widely investigated for semiconductor QDs, partly in an attempt to quench these effects. In particular, engineering of colloidal QD environment ${ }^{15,16}$ and its nanostructure ${ }^{17-19}$ provided stable nano-emitters with suppressed emission intermittency useful for quantum science and technology applications. While Purcell enhancement of a QD radiation rate through coupling of a QD transition to a plasmonic or a hybrid mode ${ }^{20-22}$ strongly quenches non radiative processes including the ones that lead to emission intermittency, we did not find any report on an effect of modified photonic environment on the QD spectral diffusion. Here, we report on the suppression of the spectral diffusion of QD-excitons incorporated in photonic cavities. It is shown that, for QD emission tuned to the resonance of the cavity, the Purcell enhancement of the exciton radiative recombination leads to reduced electrical charging of the QD environment, which, in turn, reduces the spectral diffusion range. Moreover, the experiments and modeling evidence a QD environmental self-charging due to carriers trapped in the dot itself, supporting reports on similar QD charging leading to emission intermittency in open photonic environment ${ }^{23}$.

\section{Results}

Our photonic-confined QD system presented in Fig. 1(a) consists of a single InGaAs/GaAs site-controlled QD grown in an inverted pyramid, around which a photonic crystal $(\mathrm{PhC})$ membrane cavity was defined (see Methods). We designed the QD emission wavelength to be close to resonance with the fundamental cavity mode CM0 and the first excited mode CM1 (Fig. 1(a)). Weak CM-QD coupling was verified by observing the co-polarization between the QD exciton (X) and the CM line for sufficiently small X-CM detuning ${ }^{24}$ (Fig. 1(b)). Such co-polarization is accompanied by reduced $\mathrm{X}$ lifetimes, both arising from the Purcell effect ${ }^{25}$. From the co-polarization parameters we estimate a Purcell factor of $\mathrm{F}_{\mathrm{p}} \sim 10$ for studied structures (see Methods).

Whereas most structures showed PL spectra stable over acquisition time, several samples exhibited spectral diffusion of the QD transitions (Fig. 1(c)). We attribute this spectral diffusion mainly to the fabrication-induced defects around the QDs, which can trap electric charges and induce spectral shifts via the quantum confined Stark effect and bi-exciton binding energy modifications ${ }^{26}$. It should be noticed that almost all QDs stopped diffusing spectrally if exposed to above-barrier excitation during several tens of minutes. We explain this effect by the quasi-stable states of defects or background impurities that are reached after a long exposure to photoexcitation and hence high densities of excited electron-hole pairs. For CM-X detuning of at least several meV, as in Fig. 1(c), we did not observe any effect of the CM on the spectral diffusion.

Laboratory of Physics of Nanostructures, Institute of Physics, Ecole Polytechnique Fédérale de Lausanne (EPFL), Lausanne, Switzerland. Correspondence and requests for materials should be addressed to A.L. (email: alexey. lyasota@gmail.com) 
(a)
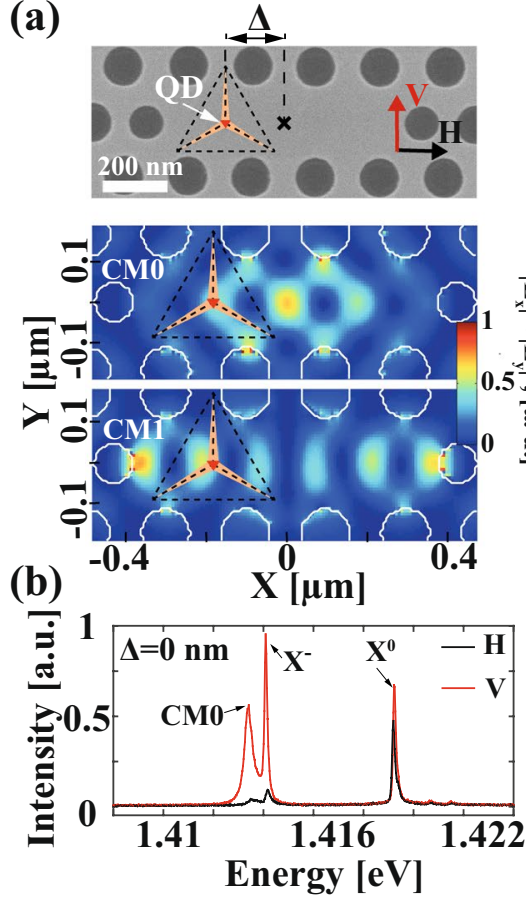

(c)

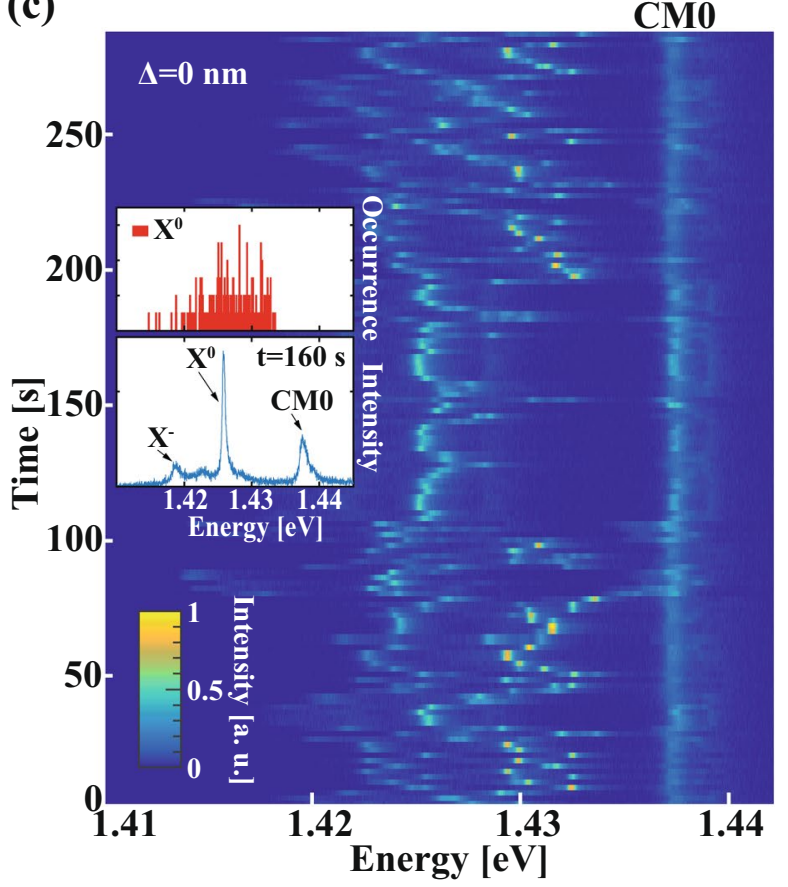

Figure 1. (a) SEM top view of the fabricated structure and computed near-field intensity patterns of modes CM0 and CM1. (b) Polarization-resolved photoluminescence (PL) spectra showing exciton-cavity mode weak coupling $(\mathrm{T}=10 \mathrm{~K})$. (c) Temporal-spectral map showing PL spectra acquired sequentially for a structure exhibiting "free" $\mathrm{X}$ spectral diffusion. Excitation power $\mathrm{P}_{\mathrm{exc}}=300 \mu \mathrm{W}, \mathrm{T}=10 \mathrm{~K}$. Insets show spectrum acquired at $\mathrm{t}=160 \mathrm{~s}$ and occurrence histogram of the neutral excitonic transition $\mathrm{X}^{\circ}$.

However, for sufficiently small CM-X detuning, we observed spectral diffusion within a restricted energy range, confined to the vicinity of the CM resonance (Fig. 2(a)). We explain this "spectral trapping" of the otherwise freely diffusing $\mathrm{X}$ transition by the combined effects of the enhanced $\mathrm{X}$ emission rate $\gamma$ due to the CM-induced Purcell enhancement at sufficiently small X-CM detuning $\delta$, and the role of the QD as a primary source of the charging for the traps in its vicinity (Fig. 2(b,c)). Similar charging mechanism leads to exciton lifetime-intensity correlations in emission intermittency of colloidal QDs ${ }^{27}$, recently also reported for InGaAs/ $\mathrm{GaAs}_{\mathrm{QD}}{ }^{23}$. In this scenario, the charging rate of the surrounding traps is proportional to the QD average occupation II with an electron-hole pair. The latter is estimated as $\Pi=\frac{1}{1+\gamma(\delta) / \mathrm{P}}$ from the steady state solution of the rate equation $\frac{d \Pi}{d t}=\mathrm{P}(1-\Pi)-\gamma(\delta) \Pi$ driving the single electron-hole dynamics in the $\mathrm{QD}$ at a population rate $\mathrm{P}$ below the $\mathrm{X}$ saturation level. The coupling of the $\mathrm{X}$ transition with the $\mathrm{CM}$ modifies the QD population depending on the $\mathrm{X}-\mathrm{CM}$ detuning $\delta=\mathrm{E}-\mathrm{E}_{\mathrm{CM}}$, where $\mathrm{E}$ and $\mathrm{E}_{\mathrm{CM}}$ are exciton and $\mathrm{CM}$ energies. The Purcell effect on the $\mathrm{X}$ recombination rate reads ${ }^{28-30}$

$$
\gamma(\delta)=\frac{\gamma_{0} \mathrm{~F}_{\mathrm{P}}\left(1+\frac{\mathrm{Q}_{\mathrm{CM}}}{\mathrm{Q}_{\mathrm{X}}}\right)}{8 \frac{\delta^{2}}{\Delta \omega_{\mathrm{CM}}{ }^{2}}+2\left(1+\frac{\mathrm{Q}_{\mathrm{CM}}}{\mathrm{Q}_{\mathrm{X}}}\right)^{2}} \eta^{2}+\gamma_{\text {leak }}
$$

where $\gamma_{0}$ and $\gamma_{\text {leak }}$ are the $\mathrm{X}$ spontaneous emission rates in the bulk (open photonic environment) and in the $\mathrm{PhC}$ gap, $\mathrm{F}_{\mathrm{P}}$ is the theoretical Purcell factor, $\mathrm{Q}_{\mathrm{CM}}=\frac{\omega_{\mathrm{CM}}}{\Delta \omega_{\mathrm{CM}}}$ and $\mathrm{Q}_{\mathrm{X}}=\frac{\omega_{\mathrm{X}}}{\Delta \omega_{\mathrm{X}}}$ are the CM and the $\mathrm{X}$ quality factors defined through the $\mathrm{X}$ and the $\mathrm{CM}$ angular frequencies, and $\eta$ is the effective CM-X spatial overlap ${ }^{25}$. Thus, the charging rate of the QD environment and the spectral diffusion of the excitonic transitions are coupled to the X-CM detuning $\delta=\mathrm{E}-\mathrm{E}_{\mathrm{CM}}$ (Fig. 2(b,c)). It should be noted that before exciton $\mathrm{X}^{0}$ started spectrally diffusing (Fig. 2(a)), its emission was almost completely quenched ${ }^{5,6}$ during the first $36 \mathrm{~s}$ due to the high electric field induced by the environmental charging. This quenching is caused by either QD ionization or by a reduced dipole moment of the QD transition.

In this picture, the charging level of the surrounding traps is proportional to the QD average occupation with an electron-hole pair, which depends on the QD pumping rate $\mathrm{P}$ and the recombination rate $\gamma$ of the QD-confined exciton. The QD recombination rate, in turn, increases with decreasing CM-X energy detuning $\delta$. Thus, the QD environment is more efficiently charged when the QD optical transition is tuned out of resonance with the CM (Fig. 2(b)). On the other hand, at resonance the electron-hole recombination rate is enhanced by the cavity Purcell effect, which reduces the average QD occupation and consequently decreases the charging rate of the traps (Fig. 2(c)). At large CM-X detuning and high charging levels, the electric field, exerted by the environment, 
(a)

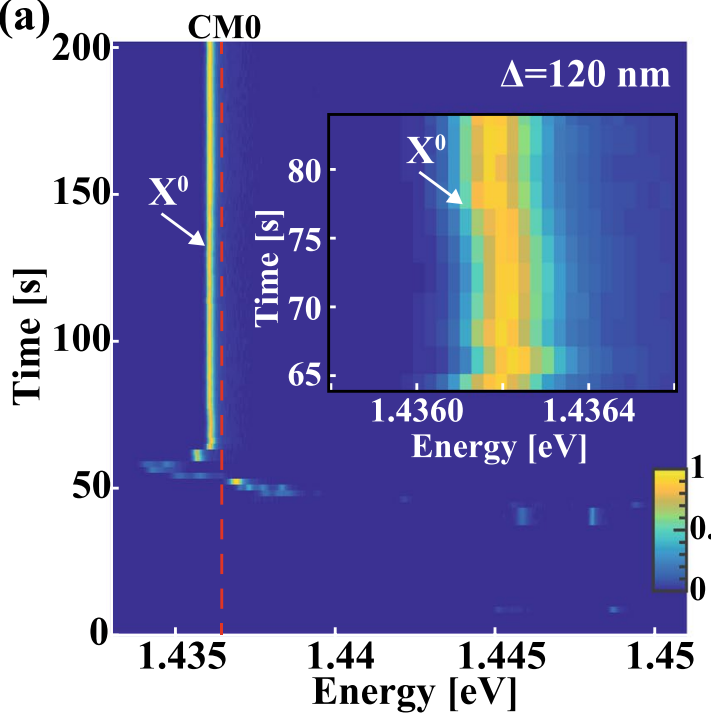

(d)

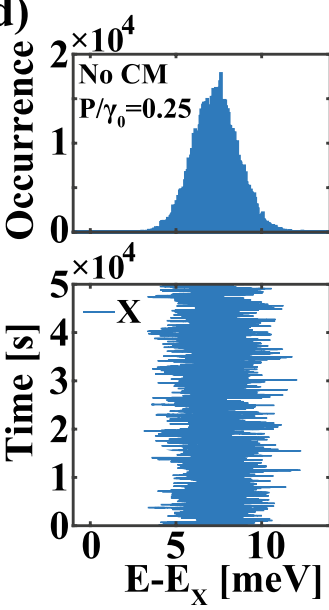

(e)

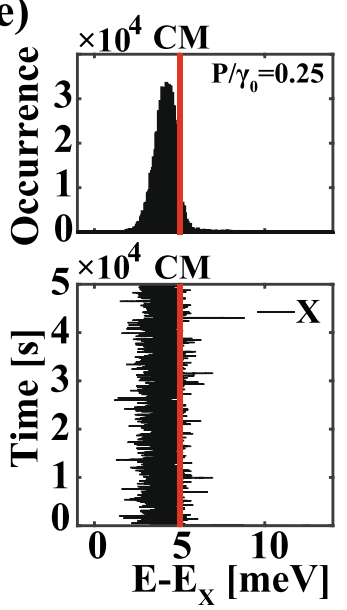

(b)

$\mathrm{X}-\mathrm{CM}$

resonance QD

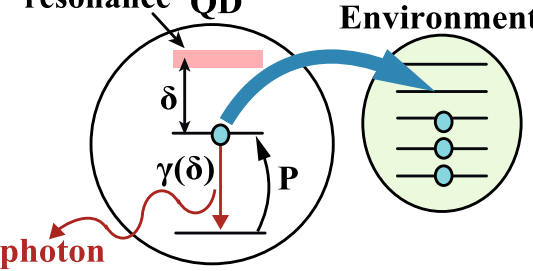

(c)

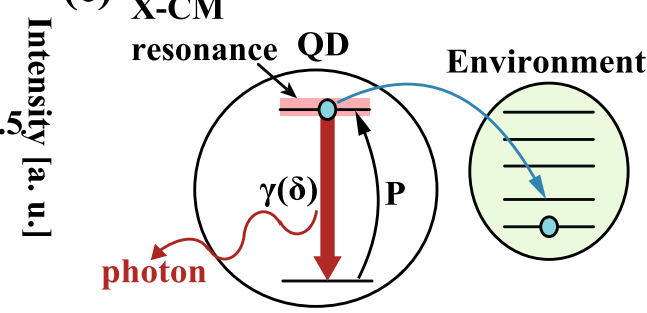

(f)

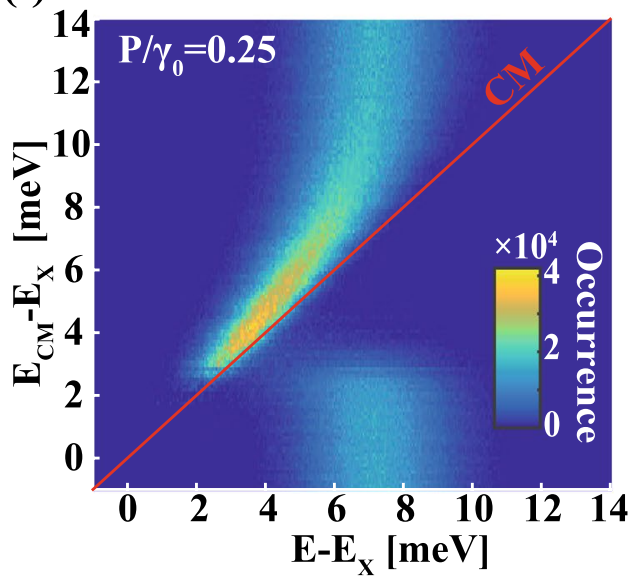

Figure 2. (a) Temporal-spectral map for an L3 PhC cavity with $\Delta=120 \mathrm{~nm}$ showing spectral trapping of the $\mathrm{X}$ by the CM mode (red dashed line). Inset shows a detail of the spectrally diffusing exciton at $t=75 \mathrm{~s}$. Excitation power $\mathrm{P}_{\mathrm{exc}}=100 \mu \mathrm{W}, \mathrm{T}=10 \mathrm{~K}$. (b,c) Schematics of the model showing the QD and charged environment for high (b) and low (c) charging rates, controlled by the X-CM detuning. Simulated X spectral diffusion: temporal trace and occurrence histogram without (d) and with (e) the CM. (f) Simulated map of the X occurrence versus relative $C M$ energy $E_{C M}-E_{X}$ for quantum dot population rate $P=0.25 \gamma_{0}$ where $E_{X}$ is unperturbed $X$ energy (see Methods for details on simulations).

shifts the X-transition provoking spectral diffusion. For small CM-X detuning, the charging level of the environment can be drastically lowered, which reduces the X-level shifts and the consequent spectral diffusion. Here, we neglected the effect of carrier losses due to traps charging on the average QD occupation. This is because such loss rate is comparable to that of spectral diffusion, that is, a few $\mathrm{Hz}$, and is at least 7-8 orders of magnitude smaller than the $\mathrm{X}$ recombination rate. The latter varies between $2 \mathrm{GHz}$ to $20 \mathrm{GHz}$, as can be extracted from the $\sim 2 \mathrm{~ns}$ and $\sim 200$ ps radiative-limited excitonic lifetime in an open photonic environment and in direct resonance with the cavity mode $(\delta=0)$, respectively.

Figure 2(d),(e) show the simulated time traces and occurrence histograms of the X-transitions as a function of the energy shift from the unperturbed (i.e., without energy shift) $\mathrm{X}$ energy $\mathrm{E}_{\mathrm{X}}$ (see Methods for details on the numerical model). Evidently, the presence of the cavity reduces the range of spectral diffusion of the $\mathrm{X}$-line, pulling the recombination energy to the vicinity of the CM. At the same time, in the presence of the CM the occurrence histogram is suppressed at the CM energy, with its peak located at one side of the CM energy. A more complete description of the occurrence distribution is shown in Fig. 2(f), which displays the simulated spectrum of the occurrence as a function of the $\mathrm{CM}$ energy $\mathrm{E}_{\mathrm{CM}}$. The occurrence assumes its maximum at either side of the $\mathrm{CM}$, a manifestation of the Purcell-enhanced recombination of excitons near the cavity resonance.

Further support to our model is provided by the dependence of the spectral diffusion pattern on the photo-excitation power $\mathrm{P}_{\mathrm{exc}}$ (Fig. 3(a,b)), measured in another device. Increasing the QD pumping rate shifts the peak occurrence of the $\mathrm{X}$ transition to higher energies and produces wider spectral diffusion range, reaching across the CM resonance. This is brought about by the higher average charging of the environment and resulting intense electric fields. Remarkably, the measured occurrence of the neutral exciton transition is depleted close 
(a)

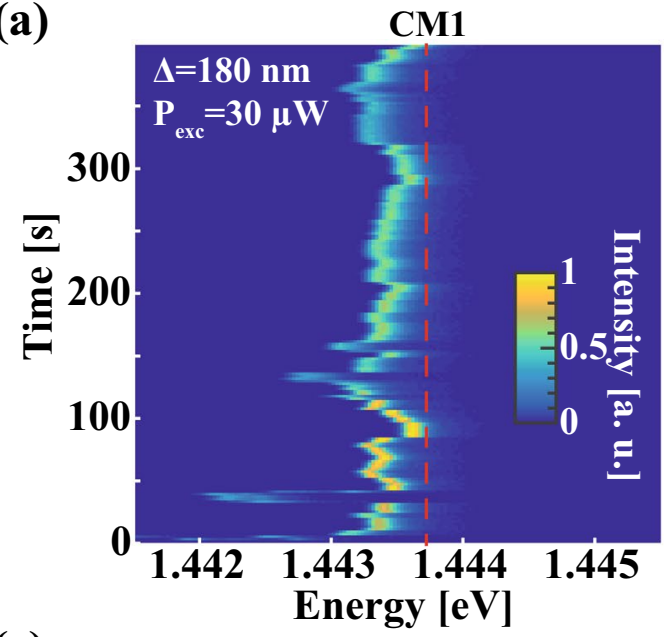

(c)

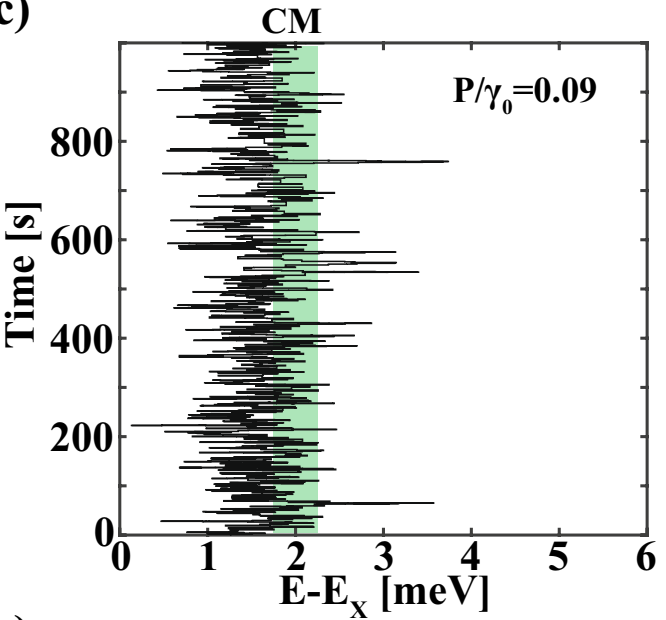

(e)

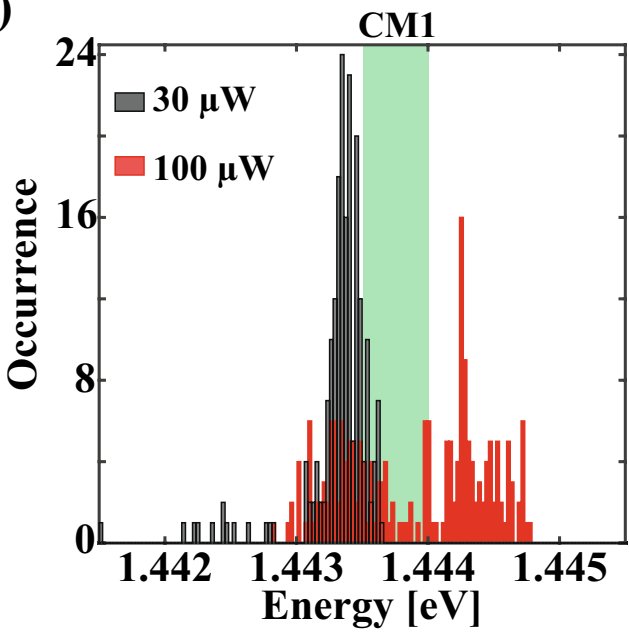

(b)

CM1

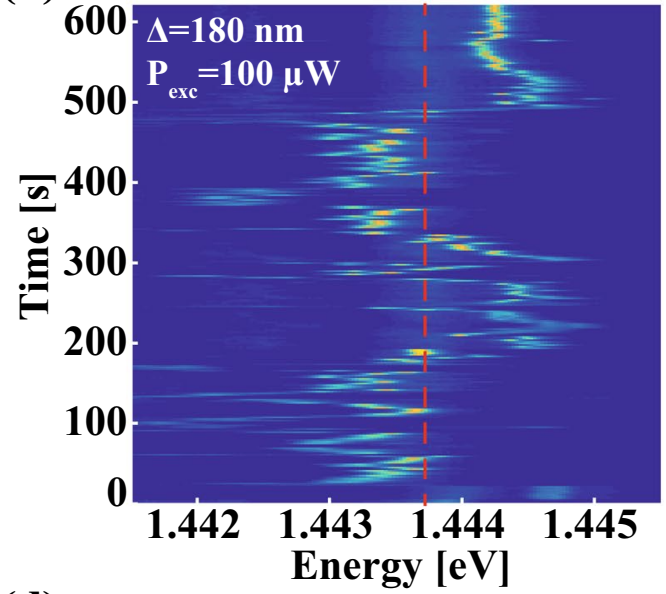

(d) CM

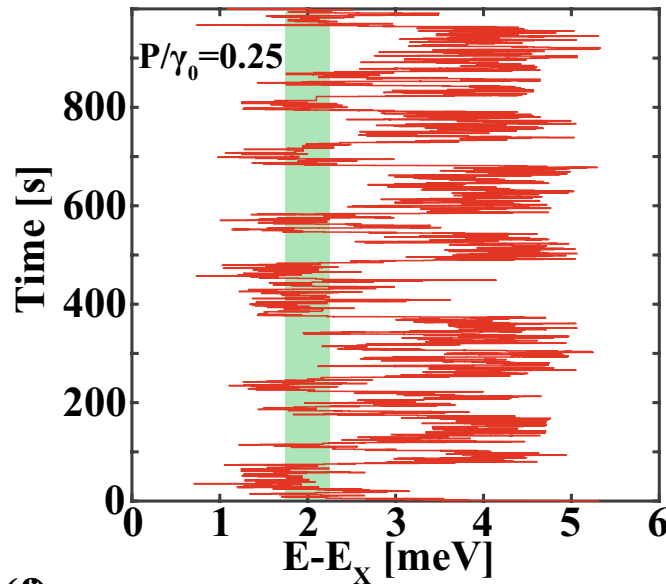

(f)

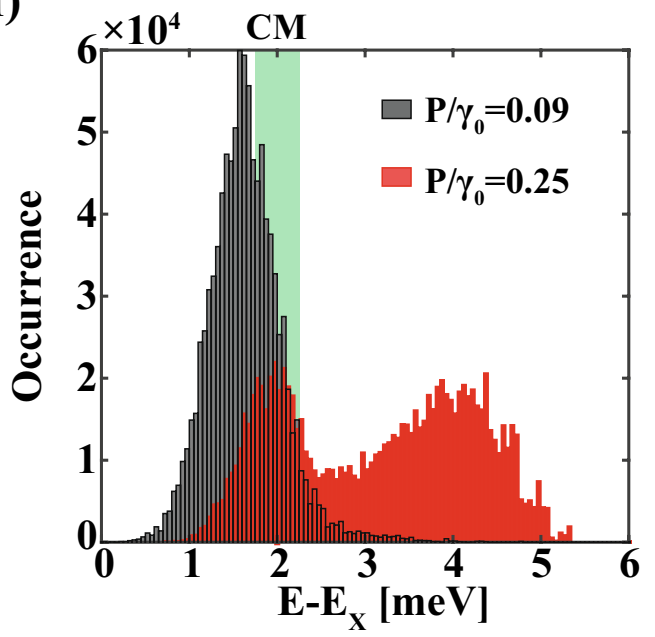

Figure 3. Measured (a,b) and simulated (c,d) excitonic trajectories for two different photo-excitation powers and QD pumping rates respectively. Corresponding measured and simulated excitonic occurrences are shown in (e) and (f). Experimental data in (a) and (b) were acquired at $\mathrm{P}_{\text {exc }}=30 \mu \mathrm{W}$ and $\mathrm{P}_{\text {exc }}=100 \mu \mathrm{W}$ excitation powers (QD in an L3 PhC cavity with $\Delta=180 \mathrm{~nm}$, mode CM1) and temperature $\mathrm{T}=10 \mathrm{~K}$. Simulated excitonic trajectories in (c) and (d) were obtained for quantum dot population rates $\mathrm{P}=0.09 \gamma_{0}$ and $\mathrm{P}=0.25 \gamma_{0}$ (See Methods for details on simulations). Same color scales used in (a) and (b).

to the CM energy, exhibiting a clear dip in the occurrence spectrum for larger excitation powers (Fig. 3(e)). Our numerical model reproduces this behavior, as reflected in both the simulated spectral diffusion traces (Fig. 3(c,d)) and spectral occurrence histograms (Fig. 3(f)). In these simulations we used 20 single-electron traps in the environment, with excitonic shifts uniformly distributed between $\mathrm{E}_{\min }=-0.06$ and $\mathrm{E}_{\max }=0.54 \mathrm{meV}$, and 
corresponding charging and discharging rates lower then $\mathrm{f}_{\max }=10 \mathrm{~Hz}$ and $\mathrm{l}_{\max }=0.1 \mathrm{~Hz}$. It can be seen that the key parameter controlling the spectral diffusion pattern is the normalized pumping rate $\mathrm{P} / \gamma_{0}$.

Additional insight into the impact of the photonic environment on the spectral diffusion is obtained by a simple analytic model for the temporal evolution of the exciton energy $E$. Assuming that the energy shift $E-\mathrm{E}_{X}$ of the excitonic transition is proportional to the total charge accommodated in the $\mathrm{QD}$ environment $\mathrm{Q}\left(E-\mathrm{E}_{X} \sim Q\right)$, we can write

$$
\frac{d E}{d t}=\frac{P \alpha}{\gamma\left(E-\mathrm{E}_{C M}\right)+P}\left(\mathrm{E}_{\text {max }}-E\right)-\beta\left(E-\mathrm{E}_{X}\right)
$$

where $\mathrm{E}_{X}$ and $\mathrm{E}_{\max }$ correspond to zero and maximum charge $\mathrm{Q}, \gamma\left(E-\mathrm{E}_{C M}\right)$ is the exciton emission rate, $\alpha$ and $\beta$ are charging and discharging rates (see Methods for details of the analytic model). For simplicity, we ignore the stochastic nature of the charging process, thus the exciton energy converges to well-defined values at infinite time. Analysis of the equation permits the determination of "stable" exciton energies, which represent the energies of highest occurrence during the spectral diffusion process.

Figure 4(a) displays the derivative $\frac{d E}{d t}$, calculated from (2), for typical parameters of our system (See Methods). The stationary points $E_{\text {stat }}$ satisfying $\frac{d^{2} E\left(E_{\text {stat }}\right)}{d t^{2}}<0$ are indicated. In an intermediate range of QD population rate $\mathrm{P}$, two points are obtained (Fig. $4(\mathrm{~b})$ ), such that the exciton transition most likely occurs above or below the CM energy. At higher QD population rates, a single stable branch exists, restoring the spectral diffusion to its pattern in the absence of a CM. As shown in Fig. 4(b), the energy at which spectral diffusion is captured depends on a particular detuning between unperturbed excitonic energy $\mathrm{E}_{\mathrm{X}}$ and $\mathrm{CM}$ energy. We corroborated this result with numerical simulations as shown in Fig. 4(d,e). Figure 4(d,e) demonstrate that the spectral diffusion 'capture' energy, as well as the range of $Q D$ pumping rates at which $Q D$ spectral diffusion is limited near $E_{C M}$, strongly depend on the $E_{X}-E_{C M}$ detuning. The free spectral diffusion of the QD transition in the absence of a cavity (Fig. 4(c)) persists at higher QD pumping rates when the $\mathrm{CM}$ is tuned closer to the unperturbed excitonic energy $\mathrm{E}_{\mathrm{X}}$ (Fig. $4(\mathrm{~d})$ ), and bunches near the CM energy for sufficiently low QD population rates (Fig. 4(e)). In all cases, the spectral diffusion in the absence of CM (Fig. 4(c)) is recovered above a threshold QD population rate, which depends on the $\mathrm{E}_{\mathrm{CM}}-\mathrm{E}_{\mathrm{X}}$ value.

Figure 4 (f) shows spectral diffusion ranges as a function of Purcell factor $\mathrm{F}_{\mathrm{p}}$ and corresponding emission rate enhancement in resonance $\gamma_{\text {res }} / \gamma_{0}$ obtained using Eq. (1). Exciton occurrence is limited to the CM side for $F_{p}$ above some threshold value $\mathrm{F}_{\mathrm{p}}^{\text {th }}$ with a full width at half maximum (FWHM) $\delta \mathrm{E}$ significantly reduced with respect to the occurrence FWHM $\delta \mathrm{E}_{0}^{\mathrm{p}}$ obtained for $\mathrm{F}_{\mathrm{p}} \approx 0$. Figure $4(\mathrm{~g})$ shows the simulated $\delta \mathrm{E} / \delta \mathrm{E}_{0}$ for 20 (set $1-2$ ) and 40 (set 3) single-electron charge traps and for various charging and discharging rates: $1_{\max }=0.3 \mathrm{~Hz}$ and $\mathrm{f}_{\max }=10 \mathrm{~Hz}$ for 'set 1 ', $\mathrm{l}_{\max }=0.1 \mathrm{~Hz}$ and $\mathrm{f}_{\max }=10 \mathrm{~Hz}$ for 'set 2', and $\mathrm{l}_{\max }=0.3 \mathrm{~Hz}$ and $\mathrm{f}_{\max }=1 \mathrm{~Hz}$ for 'set 3' (see Methods). $\delta \mathrm{E} / \delta \mathrm{E}_{0}$ reveals very similar behavior after dropping down for $\mathrm{F}_{\mathrm{p}}>\mathrm{F}_{\mathrm{p}}^{\text {th }}$ (Fig. $\left.4(\mathrm{~g})\right)$ for all used modeling parameters.

\section{Discussion}

We observed the modification of the spectral diffusion of QD excitons tuned to resonance with the modes of a photonic nano-cavity. The effect is explained by the Purcell enhancement of the QD-exciton lifetime, which, in turn, reduces the direct charging of the environment by charge released by the QD itself. We note that resonant excitation of QD excitons may avoid charging of the environment by the photoexcited carriers, but does not prevent self-charging by charges confined at the QD via the mechanism invoked in our study. Thus, the reported demonstrations $^{31,32}$ of emission of indistinguishable photons by selected QD-cavity systems using resonant QD pumping are not in contradiction with our observations. Similar to the quenching of emission intermittency in QDs placed in plasmonic nanostructures ${ }^{20,33}$, the use of confined photonic environments for quenching the spectral diffusion of nano-scale light emitters can be useful for the fabrication of novel photon sources.

\section{Methods}

The studied system consists of a single InGaAs/GaAs site-controlled pyramidal QD integrated in an L3 PhC cavity (Fig. 1(a)) fabricated on a $250 \mathrm{~nm}$ thick GaAs suspended membrane. The QD is nominally shifted by $\Delta=0 \mathrm{~nm}$, $\Delta=120 \mathrm{~nm}$ or $\Delta=180 \mathrm{~nm}$ from the center of the L3 PhC cavity (placement precision $\sim 20 \mathrm{~nm}$, confirmed by scanning electron microscopy $\left.(\mathrm{SEM})^{34}\right)$. We used structures with different quantum dot positions in this study because of the limited number of samples of the same design. However, we took care to select structures with both similar QD-CM spectral detuning and similar spectral diffusion behavior. Further details of growth and fabrication can be found $\mathrm{in}^{35-37}$.

$\mathrm{PhC}$ mode modeling. The near field intensity patterns of the CMs shown in Fig. 1 were calculated in the symmetry plane of the PhC slab using a finite-difference method.

Optical measurements. The QDs were optically excited using a continuous wave Ti:sapphire laser emitting at $730 \mathrm{~nm}$ wavelength, with the beam focused to a $\sim 1.5 \mu \mathrm{m}$ wide spot. Photoluminescence (PL) measurements were carried out with the samples placed in a He-flow optical cryostat using a spectrometer equipped with a charge coupled device (CCD) detector providing a spectral resolution of $80 \mu \mathrm{eV}$ (reported excitation powers measured before the micro-PL setup). Polarization-resolved spectra were obtained using a $\lambda / 2$ waveplate and a linear polarizer. Time-resolved PL spectra were measured with $1 \mathrm{~s}$ dead time between consecutive acquisitions. Importantly, we avoided any light exposure of the sample from the moment the cooling process started till the PL acquisition.

The reported dynamics of the spectral diffusion were obtained by measuring PL spectra with acquisition time of $\sim 2 \mathrm{~s}$ and $1 \mathrm{~s}$ dead time between consecutive acquisitions.

Cooling procedure. We avoided any light exposure of the sample before acquisition of the PL spectra in order to achieve reproducible charging conditions. Thus, keeping the sample in complete darkness before the exposure to the laser excitation is crucial for the reported experiments. 
(a)

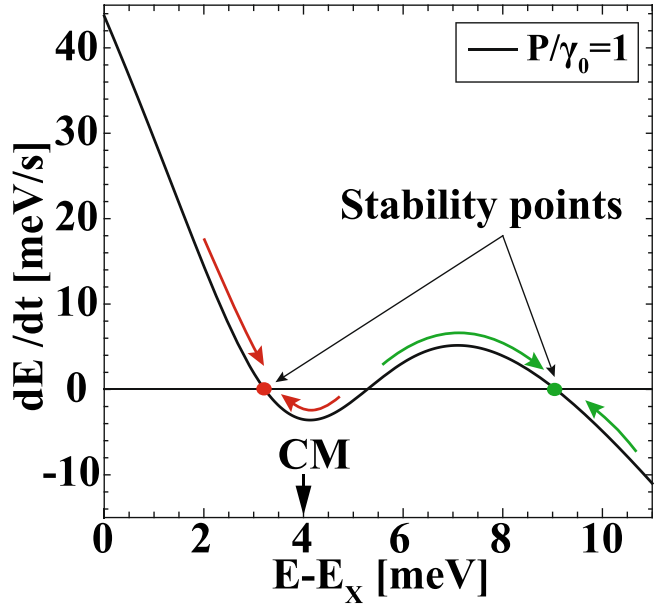

(c)

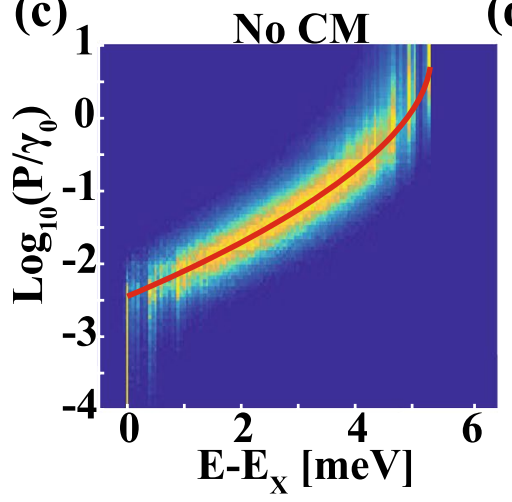

(f)

CM

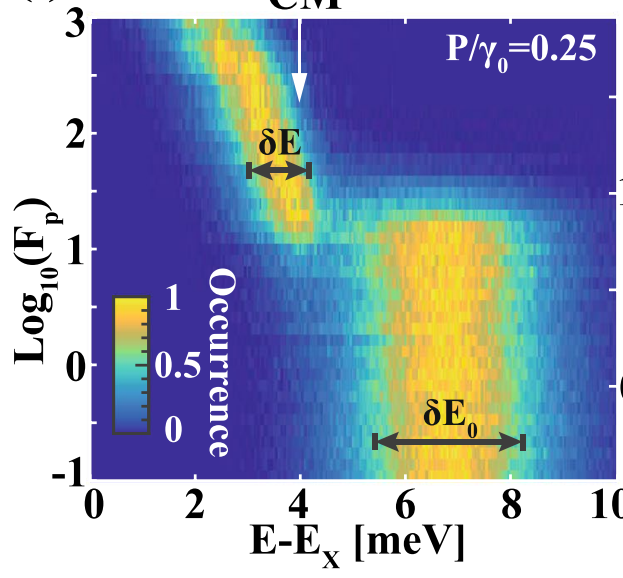

(b)

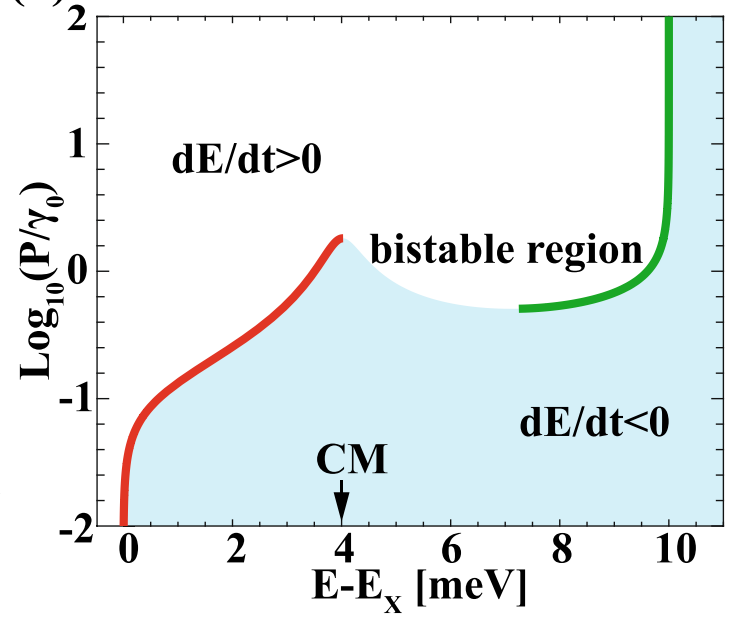

(e)

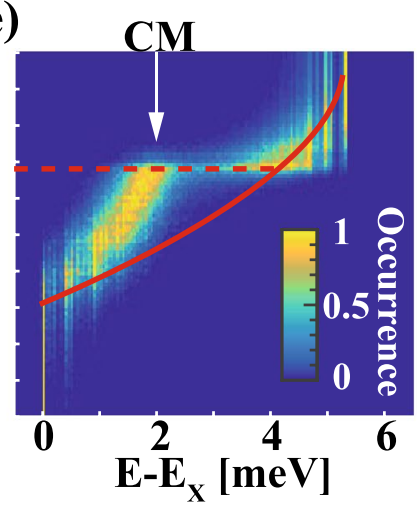

Figure 4. (a) Determination of the stability points in the exciton energy spectrum via the analytic model. (b) Calculated stability zones in the QD population rate-exciton energy plan. (c-e) Numerically simulated QD population rate- exciton energy maps of excitonic occurrence without and with CM at different energy. Solid red lines in (c-e) trace the energies of the occurrence peak without the CM. (f) Purcell factor-energy map of exciton occurrence and (g) normalized FWHM of exciton occurrence as a function of Purcell factor. Dashed red lines in (d) and (e) show the transition (normalized) power above which the occurrence peak energy recovers its values in $(\mathbf{c})$. Dashed lines in $(\mathbf{g})$ highlight $\mathrm{F}_{\mathrm{p}} \approx \mathrm{F}_{\mathrm{p}}^{\text {th }}$ values at which exciton diffusion is only partially limited to the CM side.

Charging/recombination numerical model. In the model, $\mathrm{N}$ traps, each either unfilled or filled with a single electron or hole charge, form the charged environment of the QD. We choose an occupation vector $\vec{B}=\left\{B_{i}\right\}_{i=1}^{N}$ with $B_{i}=1\left(B_{i}=0\right)$ if $i^{\text {th }}$ center is charged (not charged). Trap filling with a charge leads to either positive or negative spectral shift ${ }^{38-43}$ of the QD excitonic line $(\mathrm{X})$. Vector $\overrightarrow{\mathrm{S}}=\left\{\Delta E_{\mathrm{i}}\right\}_{\mathrm{i}=1}^{\mathrm{N}}$ describes spectral shifts provided by each of the $\mathrm{N}$ centers while it is charged. Spectral shifts are generated randomly from the uniformly distributed 
numbers in the range $\left[\mathrm{E}_{\min }, \mathrm{E}_{\max }\right]$. The total excitonic energy shift from the unperturbed excitonic energy $\mathrm{E}_{\mathrm{X}}^{0}$ is defined as $\Delta E=\overrightarrow{\mathrm{B}}^{\mathrm{T}} * \overrightarrow{\mathrm{S}}$.

The time evolution of trap occupation is driven by the system of differential equations $\frac{\partial B_{i}}{\partial t}=f_{i}\left(1-B_{i}\right)-l_{i} B_{i}$, $\mathrm{i}=1, \ldots, \mathrm{N}$ where $\mathrm{f}_{\mathrm{i}}$ and $\mathrm{l}_{\mathrm{i}}$ are charging and discharging rates of $\mathrm{i}^{\text {th }}$ trap. In our model, charging rate $\mathrm{f}_{\mathrm{i}}$ is proportional to the probability $\Pi$ of finding an electron-hole pair in the QD, that is, $f_{\mathrm{i}}=\Pi * \mathrm{~F}_{\mathrm{i}}$ where $\mathrm{F}_{\mathrm{i}}$ is the charging rate when the $Q D$ is occupied. The steady state solution of the rate equation $\frac{d \Pi}{d t}=\mathrm{P}(1-\Pi)-\gamma \Pi$ provides the QD occupation probability $\Pi=\frac{1}{1+\gamma / \mathrm{P}}$ where $\gamma$ and $\mathrm{P}$ are $\mathrm{X}$ recombination and pumping rates, respectively. Charging and discharging rates $\left\{\mathrm{F}_{\mathrm{i}}\right\}_{\mathrm{i}=1}^{\mathrm{N}}$ and $\left\{\mathrm{l}_{\mathrm{i}}\right\}_{\mathrm{i}=1}^{\mathrm{N}}$ are randomly generated from the uniform distribution in the ranges $\left[0, F_{\max }\right]$ and $\left[0,1_{\max }\right]$ tuned for better fitting of the experimental data.

The coupling of the $\mathrm{X}$ transition with the CM modifies the QD population depending on the X-CM detuning $\delta=\mathrm{E}-\mathrm{E}_{\mathrm{CM}}$, where $\mathrm{E}_{\mathrm{CM}}$ is the $\mathrm{CM}$ energy. The Purcell effect on the $\mathrm{X}$ recombination rate reads ${ }^{28-30}$

$$
\gamma(\delta)=\frac{\gamma_{0} \mathrm{~F}_{\mathrm{P}}\left(1+\frac{\mathrm{Q}_{\mathrm{CM}}}{\mathrm{Q}_{\mathrm{X}}}\right)}{8 \frac{\delta^{2}}{\Delta \omega_{\mathrm{CM}}{ }^{2}}+2\left(1+\frac{\mathrm{Q}_{\mathrm{CM}}}{\mathrm{Q}_{\mathrm{X}}}\right)^{2}} \eta^{2}+\gamma_{\text {leak }}
$$

where $\gamma_{0}$ and $\gamma_{\text {leak }}$ are the $\mathrm{X}$ spontaneous emission rates in the bulk (open photonic environment) and in the $\mathrm{PhC}$ bandgap, $F_{P}$ is the Purcell factor, $Q_{C M}=\frac{\omega_{C M}}{\Delta \omega_{C M}}$ and $Q_{X}=\frac{\omega_{X}}{\Delta \omega_{X}}$ are the CM and the $X$ quality factors defined through the $\mathrm{X}$ and the CM angular frequencies $\omega_{\mathrm{CM}}$ and $\omega_{\mathrm{X}}$ and their FWHM $\Delta \omega_{\mathrm{CM}}$ and $\Delta \omega_{\mathrm{X}}$, and $\eta$ is a CM-X spatial overlap parameter ${ }^{44}$. For our structure, we chose the Purcell factor $F_{p}=100$ and the exciton-CM overlap $\eta=0.6$ providing an emission rate $\gamma$ enhancement of $\sim 10$ times at $\mathrm{X}-\mathrm{CM}$ resonance $(\delta=0)$ for our QD-PhC cavity parameters: $\gamma_{0}=0.44 \mu \mathrm{eV}, \gamma_{\text {leak }}=0.22 \mu \mathrm{eV}, \Delta \omega_{\mathrm{CM}}=600 \mu \mathrm{eV}, \mathrm{Q}_{\mathrm{X}}=7000$ and $\mathrm{Q}_{\mathrm{CM}}=3000$. Thus, we accounted for around $90 \%$ linear emission polarization of the $\mathrm{X}$ transition observed in resonance with the $\mathrm{CM}$ for devices with $\Delta=120 \mathrm{~nm}$ and $\Delta=180 \mathrm{~nm}$.

As initial conditions, we set all traps filled at $t=0$, that is, $B_{i}=1$ for $i=1, \ldots, N$. At each computation interval $\delta \mathrm{t}=0.01 \mathrm{~s}$, charge centers lose or absorb single electrons with probabilities $\mathrm{p}_{\mathrm{i}}^{\text {loss }}=1_{\mathrm{i}} * \delta t$ and $\mathrm{p}_{\mathrm{i}}^{\text {abs }}=\frac{\mathrm{F}_{\mathrm{i}} * \delta t}{1+\gamma(\delta) / \mathrm{P}}$, shifting the $\mathrm{X}$ transition to a new excitonic energy $\mathrm{E}(\mathrm{t})$. This time evolution of the $\mathrm{X}$ energy is recorded during $t=10^{4}$ s providing modeled energy traces of $X$ transition. The occurrence histograms are obtained counting the generated energy values with $50 \mu \mathrm{eV}$ bin width. The simulated effects did not depend on the particular set of charging and discharging rates $\mathrm{F}_{\mathrm{i}}$ and $\mathrm{l}_{\mathrm{i}}$. In the model we introduced both positive and negative shifts by choosing $\mathrm{E}_{\min }<0$ and $\mathrm{E}_{\max }>0$ with in average bigger absolute values for positive shifts, that is, $\mathrm{E}_{\max }>\left|\mathrm{E}_{\min }\right|>0$. This is in accordance with the experimentally observed shifts to higher energies of the spectrally diffusing QD transitions relative to the emission energy of the non-diffusing QDs.

Analytical model of excitonic occurrence depletion. To study analytically the observed occurrence depletion, we assumed a linear dependence of the excitonic energy on the total charge Q accommodated in the QD environment: $\mathrm{E}-\mathrm{E}_{\mathrm{X}}=$ const $* \mathrm{Q}$ where $\mathrm{E}_{\mathrm{X}}$ is the excitonic energy at zero charge in $\mathrm{QD}$ environment. Assuming continuous charging and discharging of environment with rates $\alpha$ and $\beta$, we get the following differential equation driving the time evolution of the charge Q stored in the QD environment:

$$
\frac{\mathrm{dQ}}{\mathrm{dt}}=\frac{\mathrm{P} \alpha}{\gamma(\delta)+\gamma_{\text {leak }}+\mathrm{P}}\left(\mathrm{Q}_{\max }-\mathrm{Q}\right)-\beta \mathrm{Q}
$$

where $\mathrm{Q}_{\max }$ is the maximum charge that can be stored. Then, the time evolution of excitonic energy reads

$$
\frac{\mathrm{dE}}{\mathrm{dt}}=\frac{\mathrm{P} \alpha\left(\mathrm{E}_{\max }-\mathrm{E}\right)}{\frac{\mathrm{C} 1}{\left(\mathrm{E}-\mathrm{E}_{\mathrm{CM}}\right)^{2}+\mathrm{C} 2}+\gamma_{\text {leak }}+\mathrm{P}}-\beta\left(\mathrm{E}-\mathrm{E}_{\mathrm{X}}\right)
$$

where $\mathrm{E}_{\max }$ is the excitonic energy corresponding to the maximum charge accommodated in the environment, $\mathrm{C} 1=\gamma_{0} \mathrm{~F}_{\mathrm{P}}\left(1+\frac{\mathrm{Q}_{\mathrm{CM}}}{\mathrm{Q}_{\mathrm{X}}}\right) \frac{\Delta \omega_{\mathrm{CM}}{ }^{2} \eta^{2}}{8}$ and $\mathrm{C} 2=\left(1+\frac{\mathrm{Q}_{\mathrm{CM}}}{\mathrm{Q}_{\mathrm{X}}}\right)^{2} \frac{\Delta \omega_{\mathrm{CM}}{ }^{2}}{4}$ where $\mathrm{F}_{\mathrm{P}}$ is the Purcell factor, $\mathrm{Q}_{\mathrm{CM}}=\frac{\omega_{\mathrm{CM}}}{\Delta \omega_{\mathrm{CM}}}$ and $\mathrm{Q}_{\mathrm{X}}=\frac{\omega_{\mathrm{X}}}{\Delta \omega_{\mathrm{X}}}$ are the CM and the X quality factors defined through the $\mathrm{X}$ and the CM angular frequencies $\omega_{\mathrm{CM}}$ and $\omega_{X}$ and their FWHM values $\Delta \omega_{C M}$ and $\Delta \omega_{X}$, and $\eta$ is the CM-X spatial overlap. The right part of this equation can be reduced to a single fraction with a numerator being a third degree polynomial of the excitonic energy E providing either one, two or three stationary points $\mathrm{E}_{\text {stat }}$ of the time-energy curve. Among these points, stable points are provided by the condition $\frac{\mathrm{d}^{2} \mathrm{E}_{\text {stab }}}{\mathrm{dt}^{2}}<0$.

The datasets generated and analyzed during the current study are available from the corresponding author on reasonable request.

\section{References}

1. Bradac, C. et al. Observation and control of blinking nitrogen-vacancy centres in discrete nanodiamonds. Nat. Nanotechnol. 5, 345-9 (2010).

2. Ambrose, W. P. \& Moerner, W. E. Fluorescence spectroscopy and spectral diffusion of single impurity molecules in a crystal. Nature 349, 225-227 (1991).

3. Htoon, H., O'Connell, M. J., Cox, P. J., Doorn, S. K. \& Klimov, V. I. Low temperature emission spectra of individual single-walled carbon nanotubes: multiplicity of subspecies within single-species nanotube ensembles. Phys. Rev. Lett. 93, 027401 (2004). 
4. Müller, J. et al. Monitoring surface charge movement in single elongated semiconductor nanocrystals. Phys. Rev. Lett. 93, 167402 (2004).

5. Nirmal, M. et al. Fluorescence intermittency in single cadmium selenide nanocrystals. Nature 383, 802-804 (1996).

6. Robinson, H. D. \& Goldberg, B. B. Light-induced spectral diffusion in single self-assembled quantum dots. Phys. Rev. B 61, R5086-R5089 (2000).

7. Cichos, F., von Borczyskowski, C. \& Orrit, M. Power-law intermittency of single emitters. Curr. Opin. Colloid Interface Sci. 12, 272-284 (2007)

8. Pelton, M., Grier, D. G. \& Guyot-Sionnest, P. Characterizing quantum-dot blinking using noise power spectra. Appl. Phys. Lett. 85, 819-821 (2004).

9. Shimizu, K. T. et al. Blinking statistics in single semiconductor nanocrystal quantum dots. Phys. Rev. B 63, 205316 (2001).

10. Schlegel, G., Bohnenberger, J., Potapova, I. \& Mews, A. Fluorescence decay time of single semiconductor nanocrystals. Phys. Rev. Lett. 88, 137401 (2002).

11. Tang, J. \& Marcus, R. A. Mechanisms of fluorescence blinking in semiconductor nanocrystal quantum dots. J. Chem. Phys. 123, $054704(2005)$

12. Frantsuzov, P. A. \& Marcus, R. A. Explanation of quantum dot blinking without the long-lived trap hypothesis. Phys. Rev. B Condens. Matter Mater. Phys. 72 (2005).

13. Kuno, M., Fromm, D. P., Hamann, H. F., Gallagher, A. \& Nesbitt, D. J. “On”/“off” fluorescence intermittency of single semiconductor quantum dots. J. Chem. Phys. 115, 1028 (2001).

14. Verberk, R., van Oijen, A. M. \& Orrit, M. Simple model for the power-law blinking of single semiconductor nanocrystals. Phys. Rev. B 66, $233202(2002)$.

15. Hohng, S. \& Ha, T. Near-complete suppression of quantum dot blinking in ambient conditions. J. Am. Chem. Soc. 126, 1324-5 (2004).

16. Fomento, V. \& Nesbitt, D. J. Solution control of radiative and nonradiative lifetimes: A novel contribution to quantum dot blinking suppression. Nano Lett. 8, 287-293 (2008).

17. Chen, Y. et al. 'Giant' multishell CdSe nanocrystal quantum dots with suppressed blinking. J. Am. Chem. Soc. 130, 5026-7 (2008).

18. Mahler, B. et al. Towards non-blinking colloidal quantum dots. Nat. Mater. 7, 659-664 (2008).

19. Park, Y. S., Bae, W. K., Padilha, L. A., Pietryga, J. M. \& Klimov, V. I. Effect of the core/shell interface on auger recombination evaluated by single-quantum-dot spectroscopy. Nano Lett. 14, 396-402 (2014).

20. Ji, B. et al. Non-blinking quantum dot with a plasmonic nanoshell resonator. Nat. Nanotechnol. 10, 170-175 (2015).

21. Gurlek, B., Sandoghdar, V. \& Martin Cano, D. Manipulation of quenching in nanoantenna-emitter systems enabled by external detuned cavities: a path to enhance strong-coupling. ACS Photonics acsphotonics.7b00953, https://doi.org/10.1021/ acsphotonics.7b00953 (2017).

22. Peng, P. et al. Enhancing Coherent Light-Matter Interactions through Microcavity-Engineered Plasmonic Resonances. Phys. Rev. Lett. 119, 233901 (2017).

23. Hu, F., Cao, Z., Zhang, C., Wang, X. \& Xiao, M. Defect-induced photoluminescence blinking of single epitaxial InGaAs quantum dots. Sci. Rep. 5, 8898 (2015).

24. Calic, M. et al. Phonon-mediated coupling of InGaAs/GaAs quantum-dot excitons to photonic crystal cavities. Phys. Rev. Lett. 106, 227402 (2011).

25. Jarlov, C. et al. Exciton dynamics in a site-controlled quantum dot coupled to a photonic crystal cavity. Appl. Phys. Lett. 107, 191101 (2015).

26. Türck, V. et al. Effect of random field fluctuations on excitonic transitions of individual CdSe quantum dots. Phys. Rev. B 61, 9944-9947 (2000).

27. Galland, C. et al. Two types of luminescence blinking revealed by spectroelectrochemistry of single quantum dots. Nature 479, 203-7 (2011)

28. Auffèves, A. et al. Controlling the dynamics of a coupled atom-cavity system by pure dephasing. Phys. Rev. B - Condens. Matter Mater. Phys. 81, 1-10 (2010).

29. VanExter, M. P., Nienhuis, G. \& Woerdman, J. P. Two simple expressions for the spontaneous emission factor beta. Phys. Rev. A 54, 3553-3558 (1996).

30. Xu, Y., Lee, R. K. \& Yariv, A. Finite-difference time-domain analysis of spontaneous emission in a microdisk cavity. Phys. Rev. A 61, $33808(2000)$

31. Somaschi, N. et al. Near-optimal single-photon sources in the solid state. Nat. Photonics 10, 340-345 (2016).

32. Ding, X. et al. On-Demand Single Photons with High Extraction Efficiency and Near-Unity Indistinguishability from a Resonantly Driven Quantum Dot in a Micropillar, https://doi.org/10.1103/PhysRevLett.116.020401.

33. Ma, X., Tan, H., Kipp, T. \& Mews, A. Fluorescence enhancement, blinking suppression, and gray states of individual semiconductor nanocrystals close to gold nanoparticles. Nano Lett. 10, 4166-74 (2010).

34. Lyasota, A. A. Interaction between site-controlled quantum dot systems and photonic cavity structures. PhD thesis, EPFL (2017).

35. Lyasota, A. et al. Integration of multiple site-controlled pyramidal quantum dot systems with photonic-crystal membrane cavities. J. Cryst. Growth 414, 192-195 (2015).

36. Felici, M. et al. Site-controlled InGaAs quantum dots with tunable emission energy. Small 5, 938-943 (2009).

37. Mohan, A. et al. Record-low inhomogeneous broadening of site-controlled quantum dots for nanophotonics. Small 6, 1268-1272 (2010).

38. Finley, J. J. et al. Quantum-confined Stark shifts of charged exciton complexes in quantum dots. Phys. Rev. B - Condens. Matter Mater. Phys. 70, 2-5 (2004).

39. Nakaoka, T. et al. Competing influence of an in-plane electric field on the Stark shifts in a semiconductor quantum dot. Appl. Phys. Lett. 99, 81-84 (2011).

40. Nakaoka, T., Kako, S. \& Arakawa, Y. Unconventional quantum-confined Stark effect in a single GaN quantum dot. Phys. Rev. B 73, 121305 (2006).

41. Abbarchi, M. et al. Spectral diffusion and line broadening in single self-assembled GaAsAlGaAs quantum dot photoluminescence. Appl. Phys. Lett. 93 (2008).

42. Singh, R. \& Bester, G. Effects of charged defects on the electronic and optical properties of self-assembled quantum dots. Phys. Rev. $B$ 85, 205405 (2012)

43. Saito, T., Nakaoka, T. \& Arakawa, Y. Effect of lateral electric field on the transition energies of neutral and charged excitons in In 0.5 Ga 0.5 As/GaAs quantum dots. Phys. Rev. B 91, 115306 (2015).

44. Jarlov, C. et al. Effect of Pure Dephasing and Phonon Scattering on the Coupling of Semiconductor Quantum Dots to Optical Cavities. Phys. Rev. Lett. 117, 076801 (2016).

\section{Acknowledgements}

This work was supported by the Swiss National Science Foundation. We thank Dr. Giorgio Biasiol of TASC, Trieste, Italy, for the growth of the (111)B GaAs/AlGaAs membrane wafers employed. We thank Christophe Galland for critical reading of the manuscript. 


\section{Author Contributions}

B.D. and E.K. designed the samples, A.L., B.D. and A.R. fabricated the samples, C.J. did modeling of PhC structures and QD optical characterization, A.L. did optical measurements of fabricated devices and developed the theoretical model, E.K. supervised the work, A.L. and E.K. interpreted the results and wrote the article.

\section{Additional Information}

Competing Interests: The authors declare no competing interests.

Publisher's note: Springer Nature remains neutral with regard to jurisdictional claims in published maps and institutional affiliations.

(c) (i) Open Access This article is licensed under a Creative Commons Attribution 4.0 International License, which permits use, sharing, adaptation, distribution and reproduction in any medium or format, as long as you give appropriate credit to the original author(s) and the source, provide a link to the Creative Commons license, and indicate if changes were made. The images or other third party material in this article are included in the article's Creative Commons license, unless indicated otherwise in a credit line to the material. If material is not included in the article's Creative Commons license and your intended use is not permitted by statutory regulation or exceeds the permitted use, you will need to obtain permission directly from the copyright holder. To view a copy of this license, visit http://creativecommons.org/licenses/by/4.0/.

(C) The Author(s) 2019 